\title{
Perivascular epithelioid cell tumour of the bladder
}

\author{
Sarah Tarplin, ${ }^{1}$ E Charles Osterberg, ${ }^{2}$ Brian D Robinson, ${ }^{2,3}$ Michael P Herman, ${ }^{2}$ \\ James S Rosoff ${ }^{4}$
}

${ }^{1}$ Georgetown University School of Medicine, Washington, DC, USA

${ }^{2}$ Department of Urology, New York Presbyterian Hospital -Weill Cornell Medical College, New York, New York, USA

${ }^{3}$ Department of Pathology, New York Presbyterian Hospital -Weill Cornell Medical College, New York, New York, USA

${ }^{4}$ Department of Urology, Yale School of Medicine, New Haven, Connecticut, USA

Correspondence to Dr James S Rosoff, james.rosoff@yale.edu

Accepted 17 July 2014

\section{CrossMark}

To cite: Tarplin $S$, Osterberg EC, Robinson BD, et al. BMJ Case Rep Published online: [please include Day Month Year] doi:10.1136/bcr-2014205500

\section{SUMMARY}

A 39-year-old woman presented with a long history of pelvic pain and urinary urgency. Prior workup by her primary care doctor had been negative. The patient's gynaecologist ultimately referred her to a urologist following an ultrasound that revealed a possible bladder mass. MRI of the abdomen and pelvis demonstrated a $4 \mathrm{~cm}$ soft tissue lesion arising from the bladder. Cystoscopy showed an atypical mass on the anterior bladder wall, and pathological examination of the TURBT (transurethral resection of the bladder tumour) specimen revealed a perivascular epithelioid cell tumour (PEComa) with involvement of the detrusor muscle. The patient underwent a robotically assisted laparoscopic partial cystectomy. Final pathology confirmed a PEComa with negative margins. The patient had an uncomplicated postoperative course and is doing well following surgery. A surveillance cystoscopy at 6 months showed no evidence of recurrence. This case underscores the variability of clinical presentation of PEComas while proposing an appropriate method of surgical management.

\section{BACKGROUND}

PEComa refers to a diverse group of tumours with perivascular epithelioid cell differentiation. ${ }^{1}$ They are derived from mesenchymal origin and exhibit melanocytic and smooth muscle cell markers. ${ }^{1}$ The histology of these tumours was originally described as containing cells with a clear cytoplasm, epithelioid cell structure and perivascular localisation. However, the classification of PEComas has evolved into a family of tumours that includes angiomyolipoma (AML), clear cell sugar tumour (CCST), clear cell myomelanocytic tumour (CCMMT) and lymphangioleiomyomatosis (LAM). ${ }^{1}$ The entity can be associated with tuberous sclerosis in the form of AML, CCST and LAM. ${ }^{2}{ }^{3}$

PEComas occur more commonly in women, and typically occur in the fifth and sixth decade of life, although patients with tuberous sclerosis often present at a younger age. The most commonly involved organs include the lung, liver, kidneys or soft tissues, but PEComas have been reported in the bladder, skin, bone and gynaecological organs. ${ }^{1-3}$

While the histological and immunohistochemical features of PEComas are well delineated, the tumour's behaviour is less defined. Our knowledge of the entity's clinical behaviour and malignant potential is limited as it is derived from case reports, small case series and literature reviews. Most cases are benign, but rare cases of malignant PEComa have been described. Criteria have been proposed in an effort to better categorise PEComas as benign, malignant or of uncertain malignant potential. Features of malignancy include larger tumour size, hypercellularity, infiltrative growth pattern, high nuclear grade, mitotic figures $(>1 / 50 \mathrm{hpf})$, atypical mitotic figures or necrosis. ${ }^{4}$ However, these criteria were established utilising a sample of PEComas mostly of the female reproductive tract. In the urinary system, PEComas have been described more commonly in the kidney, and there are fewer reports of other locations in the urinary tract, such as the bladder. ${ }^{5}$ As such, histopathological risk factors for malignancy have been suggested for PEComas of the kidney, while it remains unclear if these can be applied to PEComas of the bladder. ${ }^{5}$

Moreover, the optimal management of patients with PEComas in kidney and bladder is poorly understood. In order to highlight the heterogeneity of this family of tumours, we report an unusual case of a young woman who presented with vague symptoms and was found to have a PEComa in an atypical anatomic location. This tumour was treated successfully with a robotic-assisted laparoscopic partial cystectomy. Here, we describe the first report of a robotic-assisted laparoscopic approach for surgical management of this rare tumour.

\section{CASE PRESENTATION}

The patient is a 39-year-old Caucasian woman with no significant medical history who presented with an 8-year history of right lower quadrant abdominal pain, which was worse with menses. She reported occasional urinary urgency, also worse with menses, but denied gross haematuria, frequency, dysuria or urinary incontinence. Gynaecological history was significant for normal Pap smears and one normal, full-term pregnancy. She denied any tobacco or alcohol use. Abdominal and pelvic examinations were unremarkable. She was previously evaluated by her primary care doctor, although prior workup was negative.

Ultimately, her gynaecologist referred her to a urologist after an abdominal ultrasound demonstrated a possible bladder lesion. Further imaging with MRI of the abdomen and pelvis showed a $4.0 \times 3.4 \times 3.3 \mathrm{~cm}$ soft tissue lesion involving the urinary bladder. The patient's urinalysis revealed sterile pyuria and her urine culture showed no bacterial growth. A voided urine cytology showed no evidence of malignant cells. The patient then underwent cystoscopy. A large anterior bladder mass was immediately visualised on entering the bladder as well as an area of trigonal leukoplakia. On bimanual examination under anaesthesia, the mass was 
palpable at the bladder neck. The patient underwent a transurethral resection of the bladder tumour (TURBT), during which the mass was debulked. However, the entirety of the tumour could not be removed due to concerns of bladder perforation. The pathology demonstrated a PEComa, confirmed by positive staining for HMB45 and smooth muscle actin, with involvement of the detrusor muscle (figure 1). Given the tumour's invasion into the detrusor muscle, the large size of the lesion, the uncertain clinical behaviour of this rare tumour and the fact that the tumour could not be completely resected transurethrally, the recommendation was made to perform a partial cystectomy. Four weeks later, the patient underwent a robotically-assisted laparoscopic partial cystectomy.

\section{INVESTIGATIONS}

The patient's urinalysis was positive for pyuria and negative for haematuria and bacteria. Her urine culture showed no growth. Urine cytology was negative for malignant cells. MRI of the abdomen and pelvis showed a $4.0 \times 3.4 \times 3.3 \mathrm{~cm}$ peripherally enhancing soft tissue lesion inferior to the urinary bladder and anterior to the cervix, possibly involving the bladder and cervix. The kidneys were unremarkable.

\section{DIFFERENTIAL DIAGNOSIS}

Urothelial carcinoma, leiomyoma, carcinoid tumour and metastasis from another site.

\section{TREATMENT}

The patient underwent a robotically assisted laparoscopic partial cystectomy. During the surgery, the mass was clearly seen arising from the anterior bladder wall into the space of Retzius. It was completely excised with grossly negative margins. The final specimen consisted of a $3.5 \times 3.0 \times 2 \mathrm{~cm}$ fleshy, yellow submucosal mass involving the muscularis propria with negative resection margins. The pathology was a tumour of perivascular epithelioid cells without evidence of malignant features.

\section{OUTCOME AND FOLLOW-UP}

The patient's postoperative course was uncomplicated. She was discharged after 1 day in the hospital tolerating a regular diet with minimal pain. Her pelvic drain was removed. After 7 days, her Foley catheter was removed following a negative cystogram. The patient is now 6 months postsurgery with a negative office cystoscopy and bladder sonogram. She will undergo quarterly office cystoscopy and bladder ultrasonography for the next year, with increasing intervals provided that there is no recurrence.
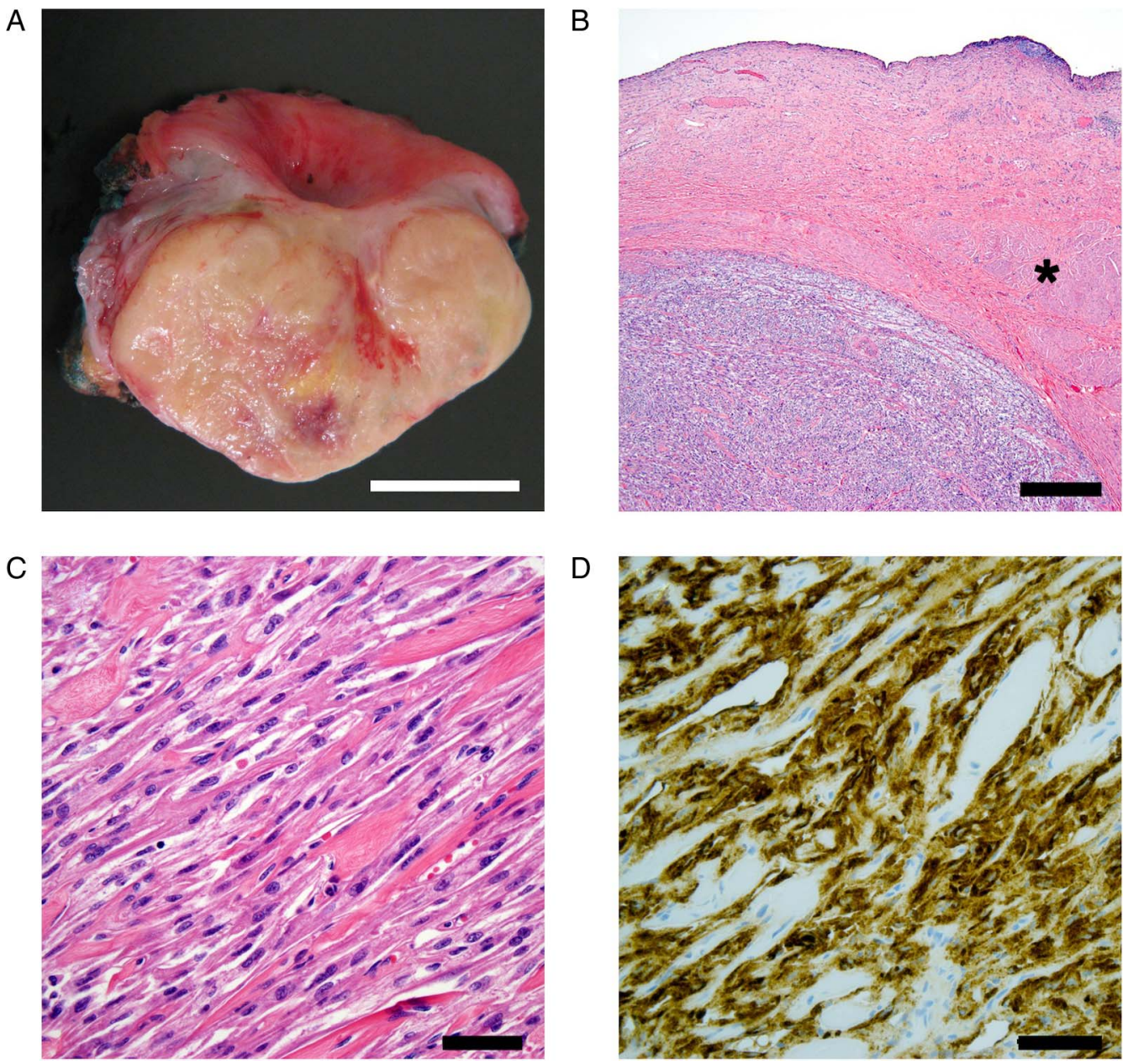

Figure 1 (A) Gross specimen demonstrating well circumscribed, fleshy, tan mass with a homogenous cut surface (line $=1 \mathrm{~cm})$. (B) H\&E stain at $\times 40$ magnification demonstrating spindle cells in a fascicular growth pattern consistent with PEComa. Asterisk marks normal detrusor muscle (line $=500 \mu \mathrm{m}$ ). (C) H\&E stain at $\times 400$ magnification demonstrating bland spindle cells with somewhat granular eosinophilic cytoplasm with no mitotic figures or necrosis (line $=50 \mu \mathrm{m}$ ). (D) Immunohistochemistry stain with HMB45 at $\times 400$ magnification (line $=50 \mu \mathrm{m}$ ). 


\section{DISCUSSION}

PEComas of the bladder are extremely rare, with fewer than 20 cases reported in the English literature over the past 10 years. In one recent review of PEComas of the urinary system, only 1 of 21 cases occurred in the bladder. ${ }^{6}$ Based on prior case reports, the mean age at diagnosis for bladder PEComa is 36, with most cases presenting between the third and fifth decade. ${ }^{7}$ There is, however, a single report of bladder PEComa in a paediatric patient-a 16-year-old Chinese girl with no known syndrome. ${ }^{8}$ Although PEComas of other anatomic sites are often associated with tuberous sclerosis, there are no reports of bladder PEComas associated with tuberous sclerosis. ${ }^{4-21}$ Unlike PEComas of other visceral organs, skin and soft tissues, PEComas of the bladder may have a male predominance. ${ }^{7}$

Based on our review of the literature, the clinical presentation appears to be variable. Some patients present with abdominal pain, as was the case with this patient, while others have clinical signs and symptoms mimicking urothelial carcinoma, such as haematuria. ${ }^{6} 7^{9-11}$ The sole paediatric case presented with vague abdominal discomfort and urinary frequency without haematuria. ${ }^{8}$ A number of reports have also described patients with lower urinary tract symptoms (including dysuria, frequency and urgency), ${ }^{6} 81012$ which is also similar to this case. This lesion may also present as an asymptomatic palpable mass or as an incidental finding on workup for another condition. ${ }^{7} 13$ Parfitt $e t a l^{10}$ described a PEComa of the bladder that presented with an enterovesical fistula. Ours seems to be the only report describing cyclical pain localised to the right lower quadrant.

Due to the relatively small number of cases and limited follow-up, it is difficult to characterise the prognosis of bladder PEComas. Folpe et $a l^{4}$ discussed criteria for classification of non-AML PEComas based on their study of soft tissue and gynaecological PEComas. 'Benign' is defined as a tumour less than $5 \mathrm{~cm}$ in size with low cellularity, low nuclear grade, mitotic rate less than 1/50 high power fields and no infiltration, vascular invasion or necrosis. 'malignant' tumours have two or more of the following features: size greater than $5 \mathrm{~cm}$, infiltrative growth pattern, high mitotic rate, pleomorphism, necrosis or vascular invasion. Having one of these features classifies the tumour as 'of uncertain malignant potential'. 7 By definition, 'malignant' features correlate with increased risk of metastasis and/or recurrence. ${ }^{4}$ This system can be applied to PEComas of the bladder. However, clinical rarity and lack of long-term follow-up limit the ability of this system to prognosticate outcomes. ${ }^{7}$ Although the majority of PEComas at other anatomic sites are benign, it is unclear whether PEComas of the urinary bladder exhibit similar natural history.

There are at least five cases of PEComas of the bladder with malignant histopathological features and three demonstrating local invasion and/or distant metastasis. $791014 \quad 15$ Reported sites of metastasis include lung, bone, sigmoid colon and gallbladder. ${ }^{9} 14$ In one report, a 55-year-old woman with a PEComa of the urinary bladder ultimately succumbed to metastatic disease only 1 year after diagnosis. Of note, this patient's tumour was found to exhibit rearrangements involving TFE3, a gene implicated in rearrangements in renal cell carcinomas and certain sarcomas. ${ }^{14}$

Although the classification scheme described above provides some guidance about prognosis, determining the optimal management for patients with bladder PEComas is challenging. The management has been variable, but local resection appears to be curative for patients with benign tumours. A few patients were managed with TURBT alone and did not require further surgical intervention. ${ }^{7} 811$ There are several reports of PEComas of the bladder treated successfully with partial cystectomy. ${ }^{7} 101213171821$ In two other cases, patients underwent radical cystectomy for metastatic tumours exhibiting aggressive clinical behaviour. ${ }^{9} 14$ Pianezza et $a l^{21}$ described in detail a laparoscopic partial cystectomy for resection of a CCMMT. While the use of robotics for the management of common bladder lesions such as urothelial carcinoma has become routine, a robotic approach to partial cystectomy for the treatment of PEComa of the bladder has not been described in prior literature. Here, we describe the first report of a robotically-assisted laparoscopic partial cystectomy for the management of a PEComa of the bladder.

The most effective treatment for locally advanced or metastatic PEComas of the bladder has yet to be determined. Adjunctive therapies such as chemotherapy, including sarcomadirected chemotherapy, immunotherapy and other agents have been utilised in this setting but their role is not clear. ${ }^{9-11} 14$ Parfitt $e t a l^{10}$ reported on the effect of adjuvant interferon- $\alpha$ therapy for a locally advanced PEComa of the bladder treated with partial cystectomy.

Here, partial cystectomy was a prudent choice because the tumour could not be fully resected safely with TURBT alone. Furthermore, the robotically assisted approach, when compared to an open approach, may have been beneficial in terms of a reduced risk of bleeding, a shorter hospital stay, improved cosmesis and a shorter recovery time. Further investigation is warranted in order to specifically characterise PEComas of the bladder in order to predict their clinical course. In addition, future studies are required to identify which patients benefit from an endourological approach versus radical surgical resection.

\section{Learning points}

- Perivascular epithelioid cell tumours are a rare family of tumours presenting in a variety of anatomic locations. In the past 10 years, there have been fewer than 20 cases of bladder PEComa reported in the English literature.

- The clinical presentation of PEComa is diverse and may mimic other benign or malignant lesions of the urinary system.

- While proposed criteria for malignancy may be helpful, the histopathological findings predicting recurrence and metastasis have not been specifically established for PEComas of the bladder.

- Robotically assisted partial cystectomy is a safe and reasonable option for management of bladder PEComas that cannot be managed by transurethral resection alone.

Contributors ST wrote and revised the manuscript. ECO developed the concept for this article and contributed to drafting of the manuscript. BDR provided pathological analysis and interpretation as well as critical revision. MPH helped to develop the concept for this article and provided critical revision at all stages. JSR provided interpretation and critical revision and coordinated the drafting of the manuscript. All the authors approved the final version.

Competing interests None.

Patient consent Obtained.

Provenance and peer review Not commissioned; externally peer reviewed.

\section{REFERENCES}

1 Auerbach A, Cassarino D. Clear cell tumors of soft tissue. Surg Pathol Clin 2011:4:783-98. 
2 Martignoni $\mathrm{G}$, Pea $\mathrm{M}$, Reghellin $\mathrm{D}$, et al. PEComas: the past, the present and the future. Virchows Arch 2008;452:119-32.

3 Walsh SN, Sanqueza OP. PEComas: a review with emphasis on cutaneous lesions. Semin Diagn Pathol 2009;26:123-30.

4 Folpe AL, Mentzel T, Lehr HA, et al. Perivascular epithelioid cell neoplasms of soft tissue and gynecologic origin: a clinicopathologic study of 26 cases and review of the literature. Am J Surg Pathol 2005;29:1558-75.

5 Nese N, Martignoni G, Fletcher CD, et al. Pure epithelioid PEComas (so-called epithelioid angiomyolipoma) of the kidney: a clinicopathologic study of 41 cases: detailed assessment of morphology and risk stratification. Am J Surg Pathol 2011;35:161-76.

6 Wang GW, Wang Y, Chen YX, et al. Perivascular epithelioid cell tumor of urinary system: a clinicopathologic analysis of 21 cases. Zhonghua Bing Li Xue Za Zhi 2012:41:443-7.

7 Sukov W, Cheville J, Amin M, et al. A Perivascular epithelioid cell tumor (PEComa) of the urinary bladder: report of 3 cases and review of the literature. Am J Surg Pathol 2009;33:304-8.

8 Yin L, Bu H, Chen M, et al. Perivascular epithelioid cell neoplasm of the urinary bladder in an adolescent: a case report and review of the literature. Ann Diagn Pathol 2012;7:183.

9 Sendo $\mathrm{H}$, Harada $\mathrm{H}$, Hasegawa $\mathrm{H}$, et al. A case of gallbladder metastasis from a malignant perivascular epithelioid cell tumor of the bladder. Nihon Shokakibyo Gakkai Zasshi 2013;110:861-8.

10 Parfitt JR, Bella AJ, Wehrli BM, et al. Primary PEComa of the bladder treated with primary excision and adjuvant interferon-alpha immunotherapy: a case report. $B M C$ Urol 2006;6:20.
11 Shringarpure SS, Thachil JV, Maya M. PEComa of the urinary bladder. Saudi J Kidney Dis Transp/ 2012;23:1032-4.

12 Huang Y, Lu G, Quan J, et al. Primary perivascular epithelioid cell tumor of the bladder. Ann Diagn Pathol 2011;15:427-30.

13 Pan CC, Yu IT, Yang AH, et al. Clear cell myomelanocytic tumor of the urinary bladder. Am J Surg Pathol 2003;27:689-92.

14 Williamson SR, Bunde PJ, Montironi R, et al. Malignant perivascular epithelioid cell neoplasm (PEComa) of the urinary bladder with TFE3 gene rearrangement: clinicopathologic, immunohistochemical, and molecular features. Am J Surg Pathol 2013:37:1619-26.

15 Weinreb I, Howarth D, Latta E, et al. Perivascular epithelioid cell neoplasms (PEComas): four malignant cases expanding the histopathological spectrum and a description of a unique finding. Virchows Arch 2007;450:463-70.

16 Abou Ghaida R, Nasr R, Shahait M, et al. Bladder perivascular epithelioid cell tumours. BMJ Case Rep 2013;2013:200153.

17 Chan AW, Chan CK, Chiu Y, et al. Primary perivascular epithelioid cell tumour (PEComa) of the urinary bladder. Pathology 2011;43:746-9.

18 Kyrou D, Staios D, Papatsoris AG. Bladder perivascular epithelioid cell tumor: a novel rare neoplasm. Urol Int 2012;88:480-2.

19 Cai JN, Shi M, Wang J. Perivascular epithelioid cell tumor, not otherwise specified: a clinicopathologic and immunohistochemical analysis of 31 cases. Zhonghua Bing Li Xue Za Zhi 2011;40:240-5.

20 Williamson SR, Cheng L. Perivascular epithelioid cell tumor of the bladder. J Urol 2011;185:1473-4.

21 Pianezza ML, Slatnik J, Evans HJ. Clear cell myomelanocytic tumour: minimally invasive treatment of a rare bladder tumour. Can Urol Assoc J 2008;2:230-4.

Copyright 2014 BMJ Publishing Group. All rights reserved. For permission to reuse any of this content visit

http://group.bmj.com/group/rights-licensing/permissions.

BMJ Case Report Fellows may re-use this article for personal use and teaching without any further permission.

Become a Fellow of BMJ Case Reports today and you can:

- Submit as many cases as you like

- Enjoy fast sympathetic peer review and rapid publication of accepted articles

- Access all the published articles

- Re-use any of the published material for personal use and teaching without further permission

For information on Institutional Fellowships contact consortiasales@bmjgroup.com

Visit casereports.bmj.com for more articles like this and to become a Fellow 